\title{
The Use of a Degradable Biomaterial to Regulate Fibrosis at the Implant-Host Interface
}

\author{
Katrina A. Harmon ${ }^{1}$, Brooks A. Lane ${ }^{2}$, John F. Eberth ${ }^{1,2}$, Michael J. Yost ${ }^{3}$, Harold I. Friedman ${ }^{4}$ and \\ Richard L. Goodwin ${ }^{5}$ \\ ${ }^{1 .}$ University of South Carolina, School of Medicine Dept. of Cell Biology and Anatomy. Columbia, SC \\ USA. \\ 2. University of South Carolina, Biomedical Engineering Program. Columbia, SC USA. \\ 3. Medical University of South Carolina, Dept. of Surgery. Charleston, SC USA \\ 4. University of South Carolina, School of Medicine, Dept. of Surgery. Columbia, SC USA. \\ 5. University of South Carolina, School of Medicine, Biomedical Sciences Dept. Greenville, SC USA.
}

Implantation of a functioning or reconstructive biomedical device into mammalian tissues is limited by the complex, foreign body-wound healing response that ensues, which eventually encapsulates the implant in a dense connective tissue. This layer can cause diverse effects including distortion and firmness of soft tissue implants, or inhibit the implant from responding to mechanical or biochemical stimuli utilize for sensing the microenvironment. The inflammatory response is initiated by a response to cellular debris and platelet aggregation by neutrophils and macrophages, signalling and activating fibroblast migration and the subsequent differentiation into myobfroblasts (MFs). Then, MFs deposit extracellular matrix around the device, effectively walling it off from host tissues prior to commencing capsule contraction. Once this response is initiated, it is irreversible; therefore, early and localized intervention in the foreign body-wound healing response offers the best opportunity to regulate the complex sequence of events. Most of the research in this area has involved alterations to the surface contour or chemical composition of implanted biomaterials; however, none of these approaches has shown intrinsically altered capsule formation. Similarly, systemic administration of drugs that affect cellular response have been ineffective or too systemically toxic. In this present study, we used implants coated with an engineered biomaterial to determine if it would alter the progression of fibrous capsule formation in a rat model of the foreign body reaction [1]. Our degradable hydrogel coating utilizes the host's innate cellular mechanisms (matrix remodelling) to deliver therapeutic agents directly to the implant microenvironment to change the trajectory of the wound healing process.

The fabrication technique that we developed to coat implants is an engineered, therapeutic collagen hydrogel to control host responses, thus improving the implant environment and implant function [2]. Numerous therapeutics can be integrated into our hydrogel including small molecules, antibodies and living cells. This flexible, degradable hydrogel allows for a multifaceted, local approach to regulate post implantation wound healing to promote successful integration and function. A three-dimensional PLA printed mold was used to fabricate hydrogel-coated silicone discs with uniform dimensions. Two therapeutic agents were incorporated into the collagen hydrogel, which was implanted submuscularly, beneath the trapezius of rats. The first agent, colchicine, is a small molecule known to inhibit tubulin polymerization and inflammatory cell migration. The other was a tagged, antibody against interleukin 8 , which is known to inhibit inflammatory cell activation and recruitment. Initial characterization indicates that the therapeutic-containing coatings had less cell invasion and remodelling of the hydrogel at the one-week time point as compared to therapeutic-free hydrogel controls (Figure 1). These controls demonstrated the expected early stages of the foreign body reaction including massive inflammatory cell invasion and degradation of the hydrogel coating. Morphometric analysis found significantly more cells 
within the hydrogel coatings of the therapeutic-free controls as compared to the Colchicine/Anti-Il-8 containing hydrogels (Figure 2). In conclusion, the present study indicates that these engineered hydrogel coatings can alter the foreign body response. Future work includes the use of a more powerful anti-inflammatory agent such as dexamethasone.

\section{References:}

[1] Soder, Brent L et al, Plastic and Reconstructive Surgery 1235 1440-1451. 2009.

[2] RLGoodwin et al, Microscopy and Microanalysis 21 (S3) 619-620. 2015.

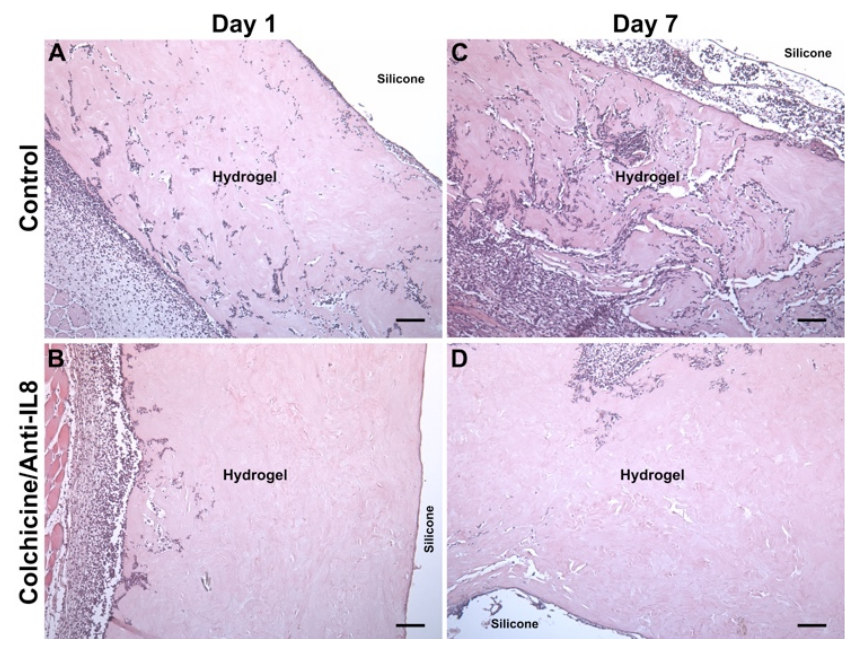

Figure 1. Therapeutic hydrogel coatings limit remodeling at one week. Less cellular infiltration was observed in hydrogel coatings containing a combination of colchicine and inhibitory IL-8 antibodies as

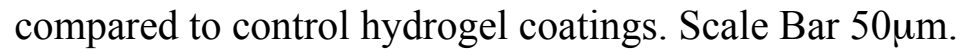

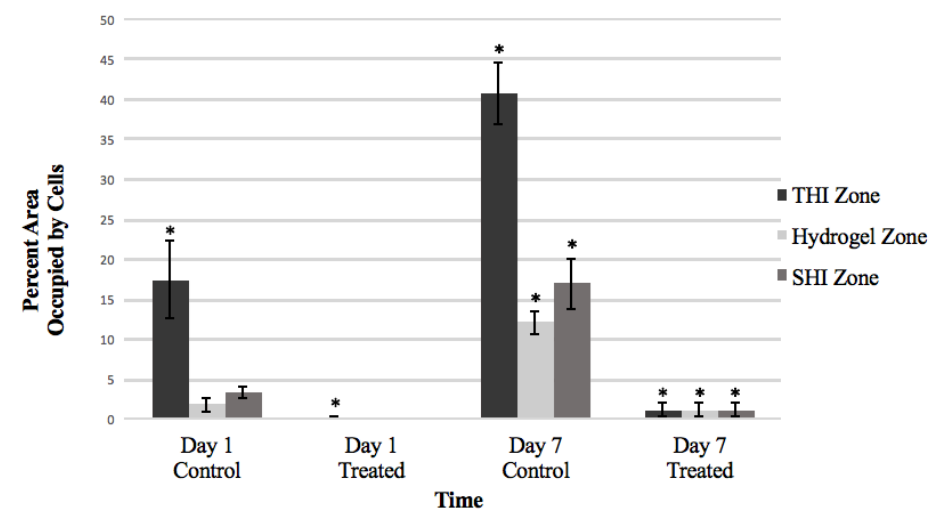

Figure 2. Morphometric analysis of hydrogel coating remodeling. $\mathrm{H}$ and $\mathrm{E}$ stained sections of the host-implant interface were analyzed for cellular density (cell area/total area) using NIH Image J. The area of the host-implant was divided into three zones (outer, middle and inner zones) and quantified. At Day 1 , a significant difference $(* p<0.05)$ was found in the cellular density at the outer, Tissue-Hydrogel Interface (THI) between the control and therapeutic containing hydrogel coated implants. Significant differences between control and treated hydrogels were found in all three zones at the Day 7 time point. $\mathrm{SHI}$ is the Silicone-Hydrogel Interface zone. 